\title{
A One-dimensional Dynamic Model of Hexagonal Telescopic Boom Considering the Deformable Cross-section
}

\author{
Yao Xie, Lei Zhang ${ }^{*}$, Yaming Tang and Aimin Ji \\ College of Mechanical and Electrical Engineering, Hohai University, Changzhou 213022, China \\ ${ }^{*}$ Corresponding author
}

Keywords: One-dimensional model, Telescopic boom, Cross-section deformation.

\begin{abstract}
In the study of telescopic boom dynamics, section deformation is often neglected. However, it has an important impact on the performance of the telescopic boom. In this paper, a one-dimensional dynamic model of hexagonal telescopic boom structure considering the cross-section deformation is established. The model considers the displacement field through the linearly superimposing of 29 basis deformation modes, which stem from the discretization of the cross-section into 8 segments. The corresponding deformation function approximated with Hermite interpolation. Involved with the principle of Hamilton, the governing equation is deduced, which is then interpolated with quadratic Lagrange for the finite element implementation. The accuracy of the new model in capturing dynamic characteristics of telescopic boom is proved by a special example.
\end{abstract}

\section{Introduction}

Telescopic boom is mostly used in engineering vehicles or other transport vehicles for a large bearing capacity in a small range and long working distance and high working height under the allowed load [1]. However, they are usually susceptible to cross-section deformation because the telescopic boom is thin. And cross-section deformation has a great influence on its performance [2]. Therefore, the effects of cross-section deformation should be considered in the dynamic modeling of telescopic boom's structure.

In recent years, many scholars have studied the section deformation of telescopic boom's structure. Li [3] studies the deformation of the web of the telescopic boom beam of the loader during operation. Jia et al. [4] discuss the relationship between stress, wrinkle and buckling of crane telescopic boom. Recently, Li et al. [5] study and summarize the influence of different cross-section shapes of telescopic boom of truck crane on its overall stability. Cheng et al. [6] establish the optimization mathematical model of telescopic arm in warehouse system. However, these models are not qualified to capture the out-of-plane and in-plane cross-section deformation of the telescopic boom completely.

The dynamic theory of flexible body system is indispensable considering the deformable cross-section of telescopic boom. Unit coupling shape function [7] and element elastic displacement field [8] are useful to describe flexible deformation, which could explain the deformation of thin-walled structures, including bending and torsion. In modal analysis, the key is to construct a or a series of [9] specific generalized eigenvalue problems to define mutually orthogonal deformation modes, such as lateral stretching mode and shear mode [10].

In this paper, a one-dimensional model for hexagonal telescopic boom considering section deformation is proposed. First, the section is discretized by interpolation method, and the displacement of discrete points is used to replace the actual displacement. Then, the similar deformation modes are linearly superposed, and the three-dimensional displacement field is reduced to one-dimensional. Using the Hamilton principle to derive the governing equation. And, the governing equations are interpolated, converted to a finite element format, and further applied in numerical examples. The rationality of the model is verified by ANSYS shell element. 


\section{Cross-section Analysis and Displacement Field}

Figure 1 shows the hexagonal telescopic boom's structure. The displacement field has three directions, including axial $u$, tangential $v$ and normal $w$, which direction along the axis of the local coordinate system is positive. Local coordinate system is defined by axial $n$, tangential $s$ and normal $z$ components. The origin of the global coordinate system $(x, y, z)$ lies at one end. There are six natural joints in the cross section, which connect adjacent walls. Since the width walls are longer than other wall lengths, nodes 7 and 8 are introduced to improve the accuracy of capturing cross-section deformation.
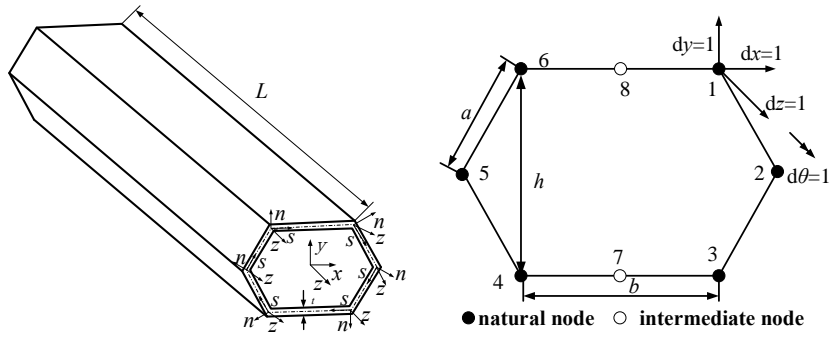

Figure 1. Global $(x, y, z)$, local coordinate system $(s, n, z)$ and discretization of the cross-sections

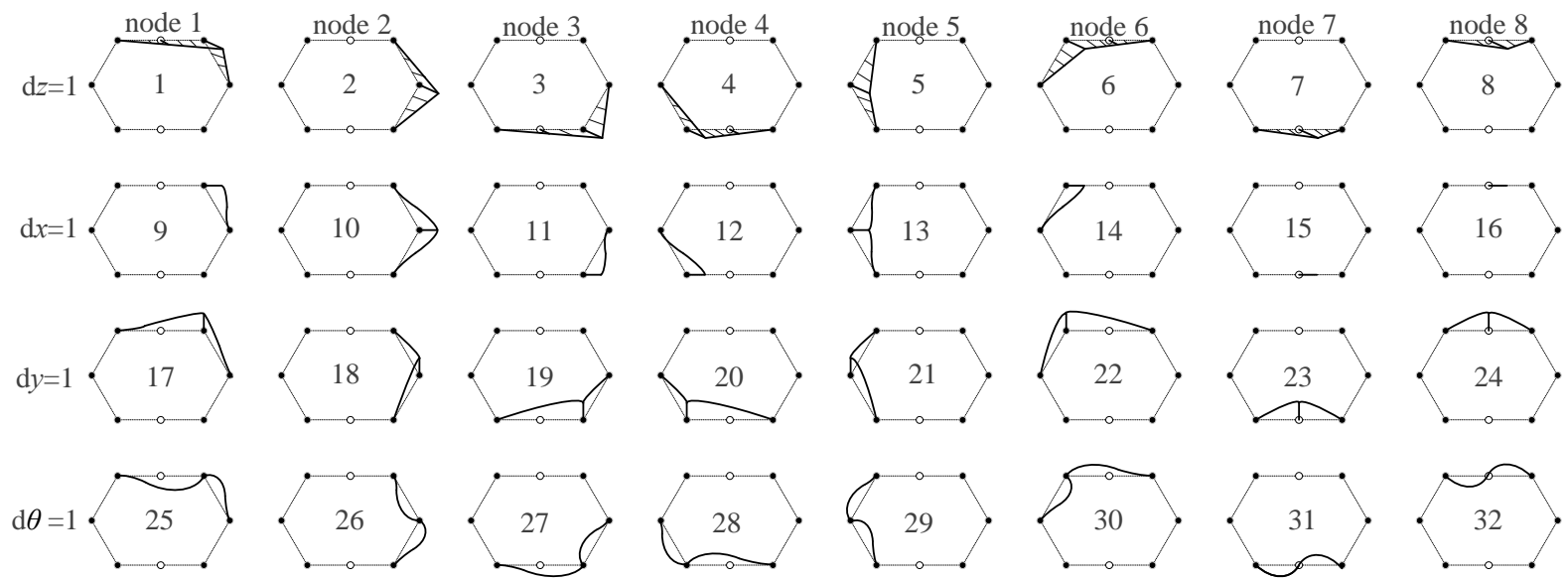

Figure 2. Basis deformation modes of the telescopic boom cross-section with 8 discretization nodes

The model discretizes the cross section by interpolation method, which includes linear Lagrange function and cubic Hermite function. Deformation modes on cross sections are described by superposition of basis functions. Considering 4 degrees of freedom, a total of 8 nodes generate 32 deformation modes.

It should be noted that not all of the above basis deformation modes (Figure 2) can be considered as the final deformation modes. To improve calculation efficiency, some special basic deformation modes can be combined into a new deformation mode. As shown in Figure 3, modes 7 and 8, 23 and 24, 31 and 32 could be combined into one deformation mode respectively, which could be observed in the actual experiment. Then the 32 deformation modes reduce to 29 deformation modes.

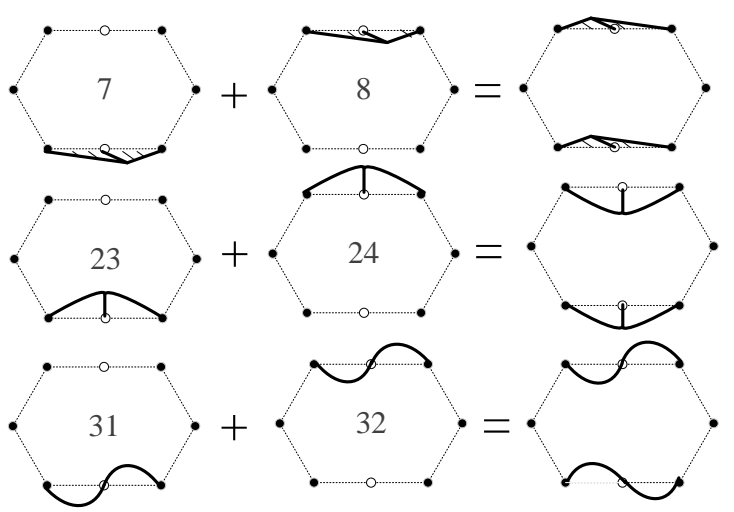

Figure 3. Assemblage of some special modes of hexagonal telescopic boom 
The deformation mode is related to the selected node and the applied displacement. The deformation function is represented by a basis function containing the variable $s$, and the deformation mode is derived in a matrix form.

The displacement variable is approximated by the basis function on the cross section [11]. The displacement field, $\mathbf{u}=[u(s, z), v(s, z), w(s, z)]^{\mathrm{T}}$ of the telescopic boom's structure is defined as:

$$
U_{z}(n, s, z)=u(s, z)-n \frac{\partial w(s, z)}{\partial z}, U_{s}(n, s, z)=v(s, z)-n \frac{\partial w(s, z)}{\partial s}, U_{n}(n, s, z)=w(s, z)
$$

The displacement field, $\mathbf{u}=[u(s, z), v(s, z), w(s, z)]^{\mathrm{T}}$ on the midline of the cross section is written as follows:

$$
\begin{aligned}
& u(s, z)=\boldsymbol{\psi}_{1} \mathbf{x}, v(s, z)=\boldsymbol{\psi}_{2} \mathbf{x}, w(s, z)=\boldsymbol{\psi}_{3} \mathbf{x} \\
& \boldsymbol{\psi}_{1}=\left[\varphi_{1}(\mathrm{~s}), \varphi_{2}(\mathrm{~s}), \ldots, \varphi_{29}(\mathrm{~s})\right], \boldsymbol{\psi}_{2}=\left[\psi_{1}(\mathrm{~s}), \psi_{2}(\mathrm{~s}), \ldots, \psi_{29}(\mathrm{~s})\right], \boldsymbol{\psi}_{3}=\left[\omega_{1}(\mathrm{~s}), \omega_{2}(\mathrm{~s}), \ldots, \omega_{29}(\mathrm{~s})\right]
\end{aligned}
$$

where $\psi_{1}, \psi_{2}$ and $\psi_{3}$ correspond to the set of basis functions. The three-dimensional displacement can be expressed as:

$$
U_{z}=\boldsymbol{\psi}_{1} \mathbf{x}-n \boldsymbol{\psi}_{3}(\partial \mathbf{x} / \partial \mathbf{z}), U_{s}=\boldsymbol{\psi}_{2} \mathbf{x}-n\left(\partial \boldsymbol{\psi}_{3} / \partial \mathbf{z}\right) \mathbf{x}, U_{n}=\boldsymbol{\psi}_{3} \mathbf{x}
$$

On the assumption of small displacement, the deformation and the corresponding stress fields are as follows:

$$
\begin{aligned}
& \varepsilon_{z z}=\boldsymbol{\psi}_{1} \frac{\partial \mathbf{x}}{\partial z}-n \boldsymbol{\psi}_{3} \frac{\partial^{2} \mathbf{x}}{\partial z^{2}}, \varepsilon_{s s}=\frac{\partial \boldsymbol{\psi}_{2}}{\partial s} \mathbf{x}-n \frac{\partial^{2} \boldsymbol{\psi}_{3}}{\partial s^{2}} \mathbf{x}, \gamma_{z s}=\frac{\partial \boldsymbol{\psi}_{1}}{\partial s} \mathbf{x}+\boldsymbol{\psi}_{2} \frac{\partial \mathbf{x}}{\partial z}-2 n \frac{\partial \boldsymbol{\psi}_{3}}{\partial s} \frac{\partial \mathbf{x}}{\partial z} \\
& \sigma_{z z}=E^{*} \varepsilon_{z z}+E^{*} v \varepsilon_{s s}, \sigma_{s s}=E^{*} v \varepsilon_{z z}+E^{*} \varepsilon_{s s}, \tau_{z s}=G \gamma_{z s}, E^{*}=\frac{E}{1-v^{2}}
\end{aligned}
$$

where $G, v$ and $E$ are the shear modulus, Poisson's ratio and material Young's modulus.

\section{Governing Equation}

By applying the Hamiltonian principle, the strain energy, the potential energy, and the kinetic energy are given by the following equations.

$$
U=\frac{1}{2} \int_{V} \boldsymbol{\varepsilon}^{\mathrm{T}} \boldsymbol{\sigma} \mathrm{d} V, U_{\mathrm{P}}=-\iiint_{V} \mathbf{U}^{\mathrm{T}} \mathbf{p} \mathrm{d} A \mathrm{~d} z, T=\frac{1}{2} \iiint_{V} \rho \partial \mathbf{U}^{\mathrm{T}} / \partial t \partial \mathbf{U} / \partial t \mathrm{~d} V
$$

where $\rho$ and $\mathbf{p}$ are the material density and the loading vector; $L, A$ and $V$ are the length of the structure, sectional area and volume. Applying the Hamiltonian principle to the governing equation.

$$
\delta \int_{t_{1}}^{t_{2}} L_{\mathrm{a}} \mathrm{d} t=0,\left.\quad \delta \mathbf{x}\right|_{t=t_{1}}=0,\left.\quad \delta \mathbf{x}\right|_{t=t_{2}}=0
$$

where $L_{\mathrm{a}}=T-U-U_{\mathrm{P}}$, and $t_{1}$ and $t_{2}$ are start and end times.

According to (5)-(8), the governing equation of the telescopic boom's structure is as follows:

$$
\iiint_{V} \delta \mathbf{x}^{\mathrm{T}} \mathbf{H}^{\mathrm{T}} \eta \mathbf{H} \frac{\partial^{2} \mathbf{x}}{\partial t^{2}} \mathrm{~d} A \mathrm{~d} z+\iiint_{V} \delta \mathbf{x}^{\mathrm{T}} \mathbf{H}^{\mathrm{T}} \mathbf{c}^{\mathrm{T}} \mathbf{E} \mathbf{c H} \mathbf{x} \mathrm{d} A \mathrm{~d} z-\iiint_{V} \delta \mathbf{x}^{\mathrm{T}} \mathbf{H}^{\mathrm{T}} \mathbf{p} \mathrm{d} A \mathrm{~d} z=0
$$

where $\mathbf{H}, \mathbf{c}$ and $\mathbf{E}$ are defined as:

$$
U_{z}(n, s, z)=\mathbf{H}_{1} \mathbf{u}, U_{s}(n, s, z)=\mathbf{H}_{2} \mathbf{u}, U_{n}(n, s, z)=\mathbf{H}_{3} \mathbf{u}
$$




$$
\boldsymbol{\varepsilon}=\mathbf{c} \mathbf{U}=\left[\begin{array}{ccc}
\frac{\partial}{\partial z} & 0 & 0 \\
0 & \frac{\partial}{\partial s} & 0 \\
\frac{\partial}{\partial s} & \frac{\partial}{\partial z} & 0
\end{array}\right] \mathbf{U}, \boldsymbol{\sigma}=\mathbf{E} \boldsymbol{\varepsilon}=\left[\begin{array}{ccc}
\frac{E}{1-v^{2}} & \frac{E}{1-v^{2}} & 0 \\
\frac{E}{1-v^{2}} & \frac{E}{1-v^{2}} & 0 \\
0 & 0 & \frac{E}{2(1+v)}
\end{array}\right] \boldsymbol{\varepsilon}
$$

\section{Finite Element Implementation}

Using quadratic interpolation functions to approximate the axial displacement field within the telescopic boom unit.

$$
\mathbf{x}=\mathbf{N X}=\left[\begin{array}{lll}
\mathbf{N}_{1} & \mathbf{N}_{2} & \mathbf{N}_{3}
\end{array}\right] \mathbf{x}
$$

where $\mathbf{X}$ and $\mathbf{N}$ are the displacement vector and the matrix of function, respectively. Equation (11) can be further written as:

$$
\mathbf{N}_{1}=\left(2 z^{2} / l^{2}-3 z / l+1\right) \mathbf{I}_{32}, \mathbf{N}_{2}=\left(4 z / l-4 z^{2} / l^{2}\right) \mathbf{I}_{32}, \mathbf{N}_{3}=\left(2 z^{2} / l^{2}-z / l\right) \mathbf{I}_{32}
$$

where $l$ and $z$ are the length of the unit and coordinate variables in a unit.

Combining (9) and (12) leads to the finite element form:

$$
\begin{aligned}
& \mathbf{m} \partial^{2} \mathbf{X} / \partial t^{2}+\mathbf{k X}=\mathbf{f} \\
& \mathbf{m}=\int_{L} \int_{A} \mathbf{N}^{\mathrm{T}} \mathbf{H}^{\mathrm{T}} \eta \mathbf{H} \mathrm{d} A \mathrm{~d} z, \mathbf{k}=\int_{L} \int_{A} \mathbf{N}^{\mathrm{T}} \mathbf{H}^{\mathrm{T}} \mathbf{c}^{\mathrm{T}} \mathbf{E} \mathbf{c H} \mathrm{d} A \mathrm{~d} z, \mathbf{f}=\int_{L} \int_{A} \mathbf{N}^{\mathrm{T}} \mathbf{H}^{\mathrm{T}} \mathbf{p} \mathrm{d} A \mathrm{~d} z
\end{aligned}
$$

where $\mathbf{m}, \mathbf{k}$ and $\mathbf{f}$ are the mass matrix, the stiffness matrix and the force matrix, respectively.

\section{Numerical Example}

To verify the accuracy of the new model, a numerical example is performed on a cantilevered hexagonal telescopic boom. Related parameters include $\rho=7830 \mathrm{Kg} / \mathrm{m}^{3}, v=0.3, E=200 \mathrm{GPa}, h$ $=0.5196 \mathrm{~m}, L=5.4 \mathrm{~m}, b=0.5 \mathrm{~m}, a=0.3 \mathrm{~m}$.

Here the new model is employed to study the free vibration of the telescopic boom of the aerial work vehicle. As shown in Table 1, the first 8 natural frequencies are obtained by using 30 proposed elements and ANSYS shell element respectively. The ANSYS model is discretized into 1663 Shell 181 4-node units with 30 in the length direction.

Table 1. Comparison of the first 8 natural frequencies of the cantilevered telescopic boom

\begin{tabular}{|c|c|c|c|}
\hline Mode & Present mode [Hz] & ANSYS shell [Hz] & Relative errors [\%] \\
\hline 1st & 19.682 & 19.541 & 0.72 \\
\hline 2nd & 24.692 & 24.544 & 0.60 \\
\hline 3rd & 107.82 & 106.87 & 0.88 \\
\hline 4th & 134.23 & 133.34 & 0.66 \\
\hline 5th & 137.10 & 141.52 & -3.22 \\
\hline 6th & 160.09 & 163.38 & -2.06 \\
\hline 7th & 164.34 & 165.95 & -0.97 \\
\hline 8th & 170.97 & 172.25 & -0.75 \\
\hline
\end{tabular}



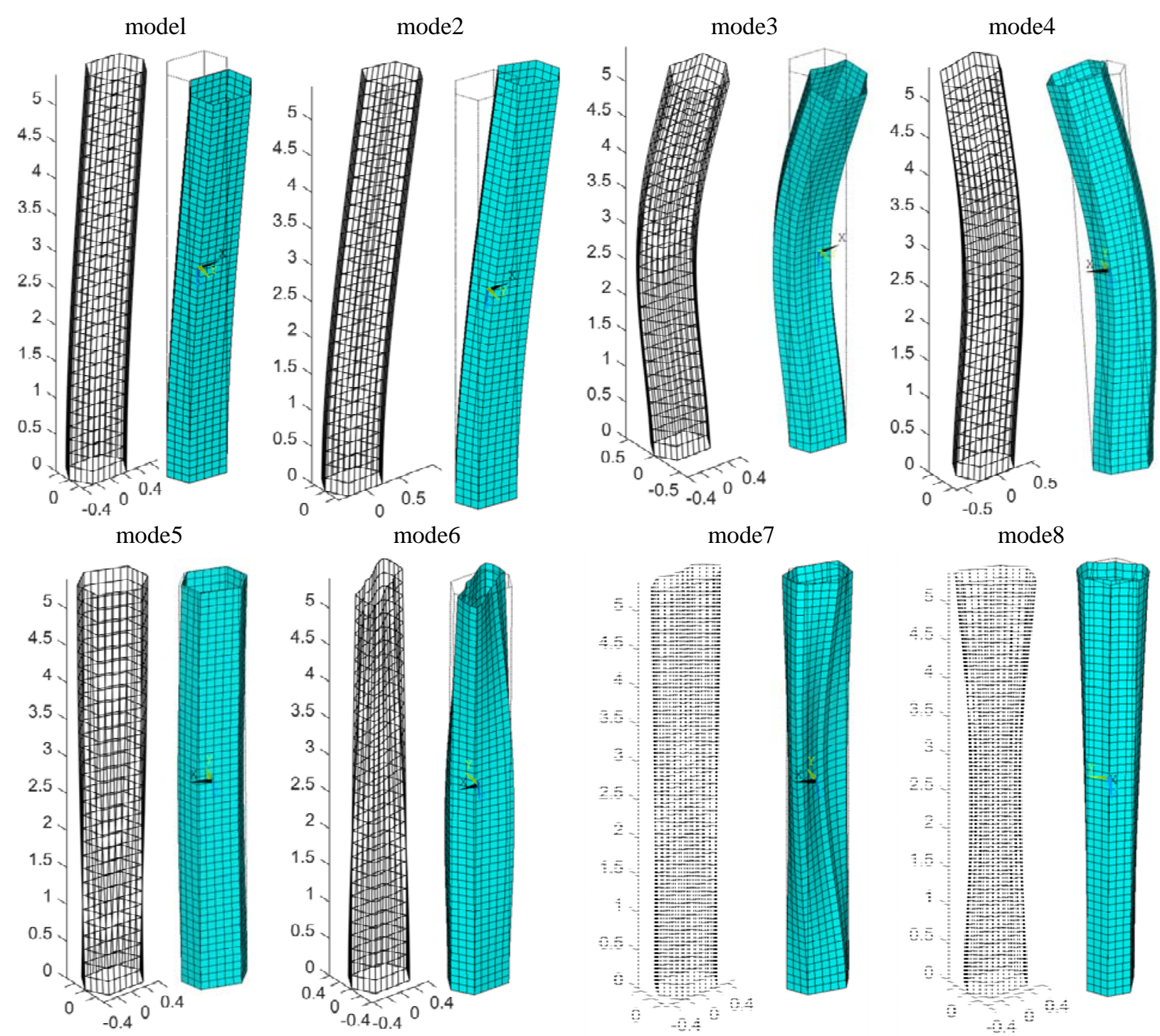

Figure 4. Comparison of modal shapes of the telescopic boom with ansys shell modal(the right ones)

As shown in Table 1, the natural frequency obtained by this model is very close to those calculated by the ANSYS shell element, and the relative error is less than 3.5\%. To further verify the mechanical properties of the telescopic boom, Figure 4 provides the first 8 modal shapes calculated by the model and ANSYS, respectively. The calculation result of the telescopic boom of the aerial work vehicle is reliable by the comparison of the modal shapes.

\section{Summary}

In consideration of the cross-sectional deformation, a new one-dimensional model for telescopic boom's structure has been proposed. In this model, approximate description of section deformation is made by interpolation at 8 discretized nodes. The deformation mode on the cross section of the telescopic boom is represented by a linear superposition of the basis functions. The governing equation is derived by using the Hamilton principle. In addition, the governing equations are interpolated, converted to a finite element format. The numerical example shows good agreement of the proposed one-dimensional model with two-dimensional shell element and proves the potential of capturing three-dimensional deformation of the hexagonal telescopic boom.

\section{Acknowledgment}

This research was financially supported by the National Science Foundation of China (Grant No. 51805144), Natural Science Foundation of Jiangsu Province (Grant No. BK20170300), and the 
Foundation of Changzhou Key Laboratory of Aerial Work Equipment and Intelligent Technology.

\section{References}

[1] J. Wang, Research on Suppression Vibration Suppression of Telescopic Arm Aerial Work Vehicle Boom [D]. Dalian University of Technology, (2016).

[2] H. Guo, H. Xi, L. Qi, and P. Feng, Telescopic boom design and finite element analysis based on Abaqus, Materials Research Vol. 1077 (2015) 215-220.

[3] J. Li, Deformation analysis and correction for girder web of telescopic arm of ship loader. Hoisting \& Conveying Machinery (2010) 87-88.

[4] J. Yao, Buckling failure analysis of all-terrain crane telescopic boom section, Engineering Failure Analysis 57(2015):105-117.

[5] R. Li, A. Ji, and L. Huang, Finite element analysis on curved cross-section telescopic boom of truck crane, Journal of Mechanical \& Electrical Engineering, 31 (2014) 1140-1143.

[6] J. Chen, Q. Liu, and Y. Liao, Structural optimization of telescopic arms in warehousing systems, Modern Machinery 1 (2016): 14-19.

[7] Z. Yan, L. Ji, J. Fan, and Y. Liu, Deformation coupling method for dynamics of flexible mechanical systems, Mechanical Engineering Journal. (2001), 37(8): 1 4.

[8] RENDEK S, BALAZ I, Distortional of Thin walled-beams, Thin-Walled Structure, (2004), 42(2):255-277.

[9] Silvestre N and Camotim D, First-order generalized beam theory for arbitrary orthotropic materials[J]. Thin-Walled Structures. (2002), 40(9): 755-789.

[10] Silvestre N and Camotim D Generalized Beam Theory Revisited: From the Kinematical Assumptions to the Deformation Mode Determination, International Journal of Structural Stability and Dynamics. (2011), 11(05): 969-997.

[11] S.J. Zhou, Finite beam element considering shear-lag effect in box girder, J. Eng. Mech. 136 (2009) 1115-1122. 